\title{
LA CONCEPCIÓN DEL "DINERO MODERNO» Y SU CONTRIBUCIÓN A UN NUEVO MARCO POLÍTICO MONETARIO-FISCAL
}

\author{
The conception of "modern money" and its \\ contribution to a new monetary-fiscal political \\ framework
}

\author{
ESTEBAN CRUZ HIDALGO \\ Universidad de Extremadura \\ evans_1987@hotmail.com

\section{FRANCISCO MANUEL PAREJO MORUNO \\ Universidad de Extremadura \\ fmparejo@unex.es}

Cómo citar/Citation

Cruz Hidalgo, E. y Parejo Moruno, F. M. (2018).

La concepción del «dinero moderno» y

su contribución a un nuevo marco político monetario-fiscal.

Revista de Estudios Políticos, 181, 167-186.

doi: https://doi.org/10.18042/cepc/rep.181.06

\section{Resumen}

La ruptura del nexo entre las políticas monetaria y fiscal, derivada de la cesión supranacional de la soberanía monetaria que han realizado los Estados, es origen de los grandes desequilibrios macroeconómicos que sacuden hoy a algunas naciones de la UEM. En este trabajo exponemos los fundamentos de la denominada teoría monetaria moderna, de los cuales subyace la urgencia de recuperar el vínculo monetario-fiscal, y consecuentemente, la necesidad de concebir las finanzas públicas de manera funcional. En contraposición a las recetas ortodoxas, que sugieren el equilibrio fiscal y la austeridad como medidas de estabilización macroeconómica, los desarrollos de la TMM contemplan el equilibrio de los sectores gubernamentales y no gubernamentales de forma conjunta, siendo deseable la expansión fiscal en períodos de recesión para el logro del objetivo de pleno empleo, sin desatender el relativo a la estabilidad de precios. 


\title{
Palabras clave
}

Dinero; política monetaria; TMM; chartalismo; hacienda funcional; códigos JEL: B19; B25; B50.

\begin{abstract}
The rupture of the nexus between monetary and fiscal policies, which derives from the supranational transfer of the monetary sovereignty by the states, is the origin of the great macroeconomic imbalances that shake today some nations inserted in the EMU. In this paper we try to show the so-called Modern Monetary Theory, from which underlies the urgency of recovering the monetary-fiscal bond, and consequently, the need of conceiving public finances in a functional way. In contrast to the orthodox recommendations, which suggest fiscal balance and austerity as macroeconomic stabilization measures, the MMT contemplates the government and non-governmental sectors balances as one, being desirable, therefore, fiscal expansion in recession periods to achieve full employment, without neglecting the one related to price stability.
\end{abstract}

\section{Keywords}

Money; monetary policy; MMT; Chartalism; functional finance; JEL codes: B19; B25; B50. 


\section{SUMARIO}

I. INTRODUCCIÓN: CONTROVERSIAS DENTRO DE LA ECONOMÍA POSKEYNESIANA. II. UNA HISTORIA ALTERNATIVA DEL DINERO: DEL METALISMO AL CHARTALISMO. III. REPENSAR LA FUNCIÓN DEL PRESUPUESTO PÚBLICO. IV. EL PLENO EMPLEO COMO OBJETIVO DE POLÍTICA ECONÓMICA. V. CONCLUSIONES: HACIA UN MARCO INSTITUCIONAL OPERATIVO. BIBLIOGRAFIA.

\section{INTRODUCCIÓN: CONTROVERSIAS DENTRO DE LA ECONOMÍA POSKEYNESIANA}

Durante las últimas dos décadas se han venido elaborando los desarrollos teóricos del enfoque conocido como "teoría monetaria moderna» (TMM) o neochartalista, integrado en la tradición heterodoxa, que comparten a grandes rasgos poskeynesianos e institucionalistas. Tales desarrollos potencian y enriquecen el marco de la «economía política radical» (entiéndase la expresión en sentido de regeneración y, en absoluto, en forma despectiva), y si bien sus elementos no son nuevos, sí han sido ignorados por buena parte de los economistas durante mucho tiempo (Fullwiller et al., 2012). Además de ayudar a comprender cómo el dinero entra en la economía, ampliando la noción de dinero endógeno ${ }^{1}$, la TMM plantea numerosas posibilidades políticas que exigen de la necesaria interrelación entre política fiscal y monetaria, y consecuentemente de las relaciones entre el tesoro y el banco central.

Desde su origen, la TMM ha recibido numerosas críticas que han sido expuestas y respondidas en Mitchell y Wray (2005), Nesiba (2013), Tymoigne y Wray (2013) y Juniper et al. (2015). Entre estas objeciones destaca la tendencia a consolidar en un solo balance las operaciones del tesoro y del banco central, lo que, además de no describir el marco institucional actual de los países desarrollados, distorsiona el entendimiento de la mecánica de compensación y liquidación en el sistema de pagos. A modo de ejemplo, la constructiva crítica hecha por Lavoie (2013) reconoce que la simplificación elaborada con la hipótesis de la consolidación no cambia la lógica en juego, puesto que, siempre y cuando el banco central demuestre determinación en el control de los tipos de interés, el resultado será el mismo.

1 Para la explicación de la noción de endogeneidad monetaria y la diferencia entre dinero endógeno y exógeno véase Cruz y Parejo (2016). 
Sobre esta crítica, Tymoigne y Wray (2013) defienden tal abstracción no solo por simplificar la exposición, sino porque mejora la comprensión de cómo el banco central está involucrado en la política fiscal y el tesoro, en la política monetaria. En el mismo sentido, Tymoigne (2016) persevera en que existe un componente vertical para el suministro de reservas que no proviene de la política monetaria, sino del tesoro. Tal y como afirma Cesaratto (2016), tras la crítica de Lavoie se ha mejorado la articulación hecha desde la TMM para la estructuración de proposiciones conflictivas, como aquella que establece que el Estado gasta antes de percibir sus ingresos fiscales o de vender sus títulos de deuda. En este sentido, este proceso no sería muy diferente de la creación monetaria ex nihilo que tiene lugar para el gasto privado, y es coherente con la ecuación de los beneficios de Kalecki de que el gasto genera los ingresos, o con la idea keynesiana de que el gasto hace posible el ahorro ${ }^{2}$.

En definitiva, los nuevos desarrollos de la TMM han abierto un nuevo panorama teórico y de debate en el ámbito monetario, pero también en el papel que desempeña la hacienda en términos macroeconómicos. Para abordar estos aspectos hemos dividido el trabajo en cinco apartados. El primero es esta introducción. En los apartados segundo, tercero y cuarto tratamos los que, a nuestro juicio, son los tres asuntos principales que se derivan de la TMM, a saber: a) la "teoría chartalista" (o del dinero del Estado); b) el marco de las finanzas funcionales propuesto por Abba Lerner, y c) la proposición de que el Estado debe actuar como empleador de último recurso, asunto que es conocido popularmente como «trabajo garantizado" o «empleo de transición». Finalmente, en el quinto apartado reflexionamos brevemente sobre las implicaciones de los desarrollos de la TMM sobre la Unión Europea y Monetaria.

\section{UNA HISTORIA ALTERNATIVA DEL DINERO: DEL METALISMO AL CHARTALISMO}

La TMM representa una comprensión del dinero diametralmente opuesta a la hipótesis metalista, la cual sugiere la existencia de un dinero-mercancía que sirve de medio de intercambio para individuos racionales, y que permite reducir los costes de transacción derivados del mismo. Esta visión ortodoxa del dinero asume su neutralidad, al considerarlo como un «velo» que simplifica y agiliza las transacciones sin afectar a las decisiones de empleo,

2 Nersisyan y Wray (2016) han concluido en la misma dirección en cuanto al carácter previo del gasto que realiza el tesoro. Para el análisis de la realidad operativa entre bancos centrales y la banca privada ver Mosler (1993). 
consumo e inversión ${ }^{3}$. Por el contrario, para la TMM el dinero es la institución más importante en la evolución hacia una «economía monetaria de producción» capitalista, y cuya aparición podemos situar hace al menos 5.000 años (Graeber, 2012).

Mientras que el mito del trueque no halla evidencia histórica alguna (Polanyi, 1976a), la TMM encuentra en las investigaciones de antropólogos, etnólogos, numismáticos y arqueólogos, entre otros científicos sociales, la base histórica y social que refuerza el planteamiento del dinero expuesto por el enfoque chartalista (Wray, 1998; Ingham, 2004a; Tymoigne y Wray, 2006). Apoyándose en el análisis del origen del dinero como un producto cultural e institucional, postula que el dinero es una unidad de cuenta designada por una autoridad pública, cumpliendo las siguientes premisas: primero, constituye una relación social, en la medida en que representa un crédito o una relación de deuda ${ }^{4}$; segundo, surgió con la aparición de las clases sociales (Bell y Henry, 2001; Henry, 2004); tercero, el valor monetario de los productos básicos en dinero nunca ha estado determinado por el valor de cambio de los metales preciosos, sino por una promesa de pago construida socialmente, esto es, una unidad de cuenta abstracta que luego es utilizada como medio de pago y para la liquidación de deudas (Ingham, 2000; Papadopoulos, 2013), y cuarto, las relaciones sociales presentan una jerarquía o pirámide de aceptabilidad, en cuya parte superior se encuentra la deuda del Gobierno (Bell, 2001).

Los chartalistas consideran que la naturaleza del dinero es intrínseca a complejas prácticas que incluyen la presencia de algún tipo de relación de dominación entre clases, lo cual es contrario a la visión de igualdad que subyace entre los participantes en la relación de intercambio del enfoque metalista. De hecho, la primera civilización en que los lazos de reciprocidad fueron alterados, apoderándose una pequeña élite del control del usufructo de la propiedad productiva, la encontramos en Mesopotamia hacia el tercer milenio a. C.

En los templos y palacios sumerios se inventó la contabilidad para llevar un registro interno de la producción y la distribución. La invención de la escritura y la administración de pesos y medidas respondían a la mayor escala y especialización de las actividades económicas. Se requería de un sistema integrado de equivalencias de precios para realizar un seguimiento de los cultivos y del adelanto de materias primas que eran abastecidas a la fuerza de

3 Los elementos de esta teoría fueron sistemáticamente expuestos en Menger (1892), y más recientemente han sido compilados en Kiyotaki y Wright (1989).

4 De hecho, la capacidad de la autoridad central de imponer una deuda tributaria en lo que ella mismo determina como dinero es el concepto clave (Wray, 2000; Tcherneva, 2002). 
trabajo, así como de los productos destinados al mercado que se suministraban a los comerciantes, quienes también conseguían para el templo aquellos materiales que no estaban disponibles en sus territorios. Para que semejante organización centralizada diese unos excedentes sistemáticos, los administradores calculaban los plazos de entrega, siempre en función de las características de la siembra y de la cosecha, de sus rendimientos potenciales, de las rentas de la tierra y de los precios pagados a los comerciantes, que eran satisfechos al regreso de sus viajes (Hudson, 2004a; 2004b).

Estos hallazgos refutan la noción de que los intercambios bilaterales producen un precio único para una mercancía, habilitándola a actuar como equivalente general. El dinero era un medio de expresar y pagar las obligaciones de los avances hechos por las instituciones públicas, es decir, una unidad de cuenta que posibilitó que las deudas se reembolsaran y los bienes se intercambiaran en función de los precios administrados por las instituciones, que disponían de una lista de precios administrados para los productos básicos que podían ser utilizados para pagar las deudas. El establecimiento de este patrón de valor abstracto sería la condición esencial para que los mercados operaran sobre la base de créditos y débitos, tomando los precios la forma de equivalencias (Polanyi, 1976b: 73).

Si bien podemos concluir que el origen del dinero está en Mesopotamia, aún faltaban en esta época los elementos fundamentales del moderno dinero-crédito, el paso que lleva a convertir el registro de deudas multilaterales llevadas en libros en formas de crédito negociables; es decir, en creación de dinero a través de préstamos bancarios. La transferibilidad de la deuda y el surgimiento de una jerarquía de aceptabilidad tuvieron una importancia crucial en el desarrollo del dinero como crédito, en forma de circulante, y la aceptación como medios efectivos de pago de estos significantes de deuda privada fueron también determinantes para el avance del capitalismo (Ingham, 2004b). Esto solo fue posible por una novedad institucional específica de esta época: la integración del endeudamiento estatal y los préstamos bancarios. En definitiva, el dinero de cuenta y la vinculación sobrevenida entre el Estado y los bancos para la creación de dinero son las relaciones institucionales clave de la economía capitalista

De acuerdo con Minsky (2008b: 258), hay una jerarquía de pasivos que describe los niveles de aceptación de las deudas emitidas por los diversos agentes. Esta jerarquía es a menudo ilustrada como una pirámide de cuatro niveles de deuda y refleja la posición de las deudas de los hogares, las empresas, los bancos y el banco central. Los bancos utilizan los depósitos del banco central como dinero, mientras que las empresas y hogares lo hacen con los depósitos bancarios. El Gobierno marca sobre las piezas de oro o papel un sello que transmite su promesa de que las aceptará para el pago de impuestos. Las 
personas forzadas a pagar impuestos deben buscar a los titulares de lo que el Gobierno reconoce como su deuda, y adquirirla mediante la venta de algún producto o servicio. Y solo cuando entregan al Gobierno sus propias representaciones de deuda es que esta deuda con él es cancelada.

El mero reconocimiento de que el emisor debe aceptar sus propios pagarés permite integrar la teoría del crédito de Innes (2004a; 2004b) con la teoría de dinero estatal de Knapp (1924). Como los pagos entre los bancos se producen con el dinero del banco central, esta relación proporciona a las promesas del banco una liquidez muy alta, como si fuesen promesas de deuda del Estado mismo, pues en última instancia lo son. El Estado debe, bien implícita o explícitamente, apoyar plenamente los pasivos o deudas bancarias, siendo también el último garante de la liquidez de estas deudas a través de su banco central, lo que implica que sean consideradas tan solventes que nadie pida que les sean reembolsadas. La aceptabilidad general de tales promesas les otorga un valor, resultante de la utilidad que tienen para liberarse de las obligaciones impuestas por la autoridad. La deuda privada emitida por los bancos podría ser aceptada para la liquidación de operaciones, pero es la utilización de la prerrogativa del Estado para declarar que es dinero la motivación por la cual la deuda del banco se convierte en dinero.

La unidad o dinero de cuenta es legítimamente elegida por el Estado, y esto va más allá de las leyes de curso legal (Wray, 2000a). En función de la jerarquía aquí presentada el dinero puede ser siempre considerado como una deuda del Estado, aunque no sea emitido propiamente por él, e incluye como dinero todo lo que sea aceptado como pago para cancelar las obligaciones con las que el Estado grava a los agentes a través de su poder coactivo.

En definitiva, la autoridad no solo regula lo que es y las medidas que servirán como dinero de cuenta, sino que además de imbuir transferibilidad al crédito privado también utiliza los impuestos como vehículo para el lanzamiento de nuevas monedas con el fin obtener de los individuos transferencias de recursos reales, obligando a pagar con estas monedas la liquidación de las obligaciones que impone. Entender que el dinero es una «criatura del Estado» (tomando la expresión acuñada por Georg Friedrich Knapp) conduce lógicamente al enfoque de Lerner sobre las finanzas funcionales.

\section{REPENSAR LA FUNCIÓN DEL PRESUPUESTO PÚBLICO}

Al hilo de lo expuesto por la teoría chartalista, la concepción del moderno dinero-crédito implica: por un lado, que un Estado no puede ser insolvente en el dinero que él mismo emite y en el que es capaz de implantar obligaciones a los agentes privados en el ámbito de su soberanía, y por el otro, que esta 
característica es esencial para garantizar el crecimiento económico de la sociedad capitalista. Partiendo de ello, la TMM analiza las distintas opciones de política fiscal y monetaria que existen en función de si un Gobierno es soberano monetariamente o no, concretando el concepto de soberanía monetaria como el poder de emitir su propia moneda y dejarla flotar (Wray, 2014).

A nivel agregado, siguiendo el principio contable de partida doble (por cada activo financiero existe un pasivo financiero igual que lo compensa), no puede haber ahorro neto de activos financieros del sector no gubernamental sin que el sector gubernamental acumule déficits públicos ${ }^{5}$. La ampliación del modelo para incluir el sector exterior no provoca ninguna diferencia fundamental en el análisis, por tanto, los sectores privados nacionales y extranjeros se pueden consolidar en el sector no gubernamental sin pérdida de visión analítica. Un Gobierno soberano a través de su gasto es la única entidad que puede proporcionar al sector privado los fondos para acomodar cualquier deseo de obtener activos financieros netos, y consecuentemente para eliminar el desempleo (Juniper y Mitchell, 2008). El gasto deficitario de un sector es el que genera el excedente o superávit del otro, de modo que la única situación en que el sector público acumula superávits es cuando los agentes privados están endeudándose, lo cual evidencia una situación potencialmente inestable en cuanto las expectativas de los agentes cambian. En definitiva, el déficit del Gobierno financia el ahorro del sector no gubernamental, pues el déficit presupuestario proporciona los ingresos que permiten al sector no gubernamental tener superávit (Wray, 2009).

Las proposiciones de la TMM chocan con la ortodoxia y llevan a rechazar la tradicional política económica basada en el equilibrio presupuestario en materia fiscal y la independencia del banco central para el control de la inflación. En su lugar, constituyen un marco operativo para la política fiscal y monetaria que implica que los Estados con control soberano sobre sus monedas no se enfrentan a ninguna restricción financiera operativa ni tienen la obligación de tomar prestado, vender bonos o recaudar impuestos para financiar sus gastos. Es lo que Lerner (1943) definió como «hacienda funcional» o «finanza funcional».

La capacidad del Estado soberano de emitir moneda y usarla para comprar bienes y servicios de la población denota su poder. Su capacidad para imponer tributos, crear y destruir dinero, comprar y vender bonos o administrar los

5 Se puede expresar con esta ecuación: saldo balance sector privado + saldo balance sector público + saldo balance exterior $=0$. Si se suman los déficits en los que incurren uno o más sectores, el resultado debe ser igual a los superávits en los que incurre el resto (Wray, 2015: 5). 
precios que paga constituye un menú de instrumentos con los que puede buscar activamente la plena ocupación y la estabilidad del valor de la moneda (Forstater, 1999).

El nivel de empleo depende del volumen de gasto que hay en la economía, lo que determina la cantidad de producto que puede venderse. Es por ello que desde la TMM se presenta el desempleo como un fenómeno monetario, proponiéndose un marco institucional que integre mecanismos que permitan promover medidas para alcanzar y mantener el ritmo adecuado de gasto monetario al nivel de pleno empleo. Tanto el volumen de las existencias de dinero como el volumen de la deuda pública no son más que la consecuencia de los pasos que habrá que dar para impedir que la cuantía del gasto no sea ni demasiado pequeña para producir desempleo ni demasiado grande para dar lugar a un aumento continuado del nivel de precios (Lerner, 1957: 112-113).

La hacienda funcional como guía de política económica es la sustitución de una regla por otra. No establecer un límite arbitrario a la deuda pública no significa que la deuda pueda aumentar indefinidamente. En vez de mantener el gasto público en el nivel en que es igual a la recaudación de impuestos, se impone al Gobierno la obligación de mantener el gasto en el nivel para el cual la demanda total del sistema no origina ni inflación ni deflación (Lerner, 1957: 329). Debe quedar claro entonces que el nivel de déficit no es discrecional, sino que responde a las decisiones de ahorro del sector privado.

Por suerte, muchas de las enseńanzas y proposiciones esbozadas por Lerner sobre la llamada hacienda funcional han sido desarrolladas y ampliadas por economistas de la TMM, evidenciando cómo la política fiscal tiene más que ver con la cantidad de dinero de lo que por lo general se reconoce, siendo, en gran medida, como ya hemos señalado, no discrecional. Mosler (19971998) y Bell (2000) inciden en este punto, mostrando cómo opera la hacienda funcional para mantener el tipo de interés objetivo fijado por el banco central a través de la adición o drenaje de reservas del Gobierno ${ }^{6}$. Su análisis revela que todo el gasto del Gobierno es financiado mediante la creación de dinero por el banco central, y que la venta de bonos y los impuestos son medios alternativos por los que se pueden drenar las reservas o destruir dinero con el fin de evitar que la tasa de interés a un día caiga a cero. Por tanto, no tienen nada que ver con la función habitual que se les asigna.

Lerner no veía cómo podía compaginarse la hacienda funcional con la idea de cebar la bomba de la demanda agregada a través de incentivar el gasto

6 La segunda ley de la hacienda funcional de Lerner plantea que el Estado solo ha de pedir prestado cuando sea deseable que el público posea menos dinero y más bonos del Estado, o al revés, con el objetivo de mantener el tipo de interés objetivo (Lerner, 1943). 
privado. La oposición a la vía de cerrar la brecha de producción para alcanzar el pleno empleo en Keynes ha sido estudiada por Tcherneva (2012), destacando el papel que este atribuía a los factores subjetivos ${ }^{7}$, y su rechazo a los cálculos de la producción potencial a precios corrientes, una metodología que es usada hoy. Tcherneva (2014) detalla cómo el mecanismo de goteo hacia abajo que actúa en las políticas de inflar la demanda, confiando en el efecto multiplicador, nunca llega lo suficientemente lejos para ofrecer un empleo a todo aquel dispuesto a trabajar, especialmente a los trabajadores de baja cualificación, no existiendo procesos de mercado endógenos que eliminen el desempleo. Por el contrario, la idea de Keynes, y al parecer también de Lerner, es lograr el pleno empleo desde un enfoque de demanda dirigida destinado a cerrar la brecha de mano de obra.

De acuerdo con lo anterior, el concepto de hacienda funcional proporciona un marco institucional adecuado para comprender el papel de la política presupuestaria y sus instrumentos en el contexto del control del nivel de gasto total necesario para lograr el pleno empleo y la estabilidad de precios, juzgando las medidas fiscales por su función en la economía. Además, la hacienda funcional puede ser útil también para afrontar cuestiones relativas al logro del pleno empleo a través de diversas fórmulas de gasto directo por parte del Estado que simultáneamente le faculten para lograr otros fines deseados socialmente, y cuya puesta en marcha no tiene por qué ajustarse a una rentabilidad estrictamente económica. Entre estos fines podemos citar la mejor equidad en la distribución, la sostenibilidad del medio ambiente, la modificación de las relaciones de género y de las relaciones laborales, o también, como sugieren Espósito y Mastrommateo (2015), la posibilidad de «implementar políticas capaces de aumentar la eficiencia y la productividad e incrementar el capital humano y tecnológico de la nación». El propósito que subyace a esta iniciativa es el de vincular pleno empleo e innovación, obligando a las empresas a competir a partir de la creación de valor añadido y no en la reducción de salarios.

Todas estas metas han sido abordadas desde la TMM mediante el planteamiento de diversos elementos que podrían guiar la puesta en marcha de diversos programas de garantía de empleo incluidos en un "plan de trabajo garantizado", como veremos a continuación en el apartado 4, proporcionando así una respuesta estructural y directa a diversas cuestiones sociales y retos urgentes a afrontar, que en nombre de la eficiencia económica se desechan.

\footnotetext{
Estos factores subjetivos son tres: la eficiencia marginal del capital, la propensión marginal a consumir y la eficiencia marginal del dinero. Su impacto en la economía depende de las expectativas de futuro de las empresas, las preferencias personales de los individuos y el apetito por la liquidez de la comunidad en su conjunto.
} 


\section{EL PLENO EMPLEO COMO OBJETIVO DE POLÍTICA ECONÓMICA}

La cuestión fundamental que se desprende del análisis de los balances sectoriales es que el sector no gubernamental depende del Gobierno para obtener fondos para su ahorro de activos financieros netos deseado y el pago de impuestos. Para obtener estos fondos, tal y como describe la teoría chartalista, los agentes no gubernamentales ofrecen bienes y servicios reales a la venta a cambio del dinero deseado, servicios que incluyen la oferta de mano de obra desempleada. Ello conduce a la obvia conclusión de que el desempleo aparece cuando el gasto del Gobierno no es el suficiente para acomodar la necesidad de pagar impuestos y el deseo de ahorrar dinero de los agentes privados.

El plan de trabajo garantizado (PTG) es el mecanismo presentado desde la TMM para alcanzar el pleno empleo a través de la oferta de un salario fijo a cualquiera dispuesto y capaz de trabajar en una serie de programas puestos en marcha por el sector público. El PTG invierte la causalidad y el énfasis que defiende la ortodoxia neoclásica con el tradicional intercambio entre inflación y desempleo representado en la curva de Philips (o la NAIRU). En vez de establecer la estabilidad de precios como una precondición crucial para un crecimiento sostenido de la actividad económica y del empleo (usando el desempleo como mecanismo de control de la inflación), el PTG garantizaría la estabilidad de precios a través de la plena ocupación de los recursos humanos (Mitchell, 1998). Tal y como lo definen Mitchell y Mosler (2002), se trata de cambiar un paradigma basado en el "gasto sobre una regla de cantidad» por el "gasto sobre una regla de precios», permitiendo a las fuerzas del mercado determinar la cantidad total del gasto público.

Según sostiene Wray (2000b), ajustar el salario del PTG al salario mínimo interprofesional podría provocar en todo caso una única subida de precios, motivada por el ajuste de todos los precios restantes a este patrón dado de demanda relativa. Además, el salario básico uniforme ofrecido por el plan de garantía de empleo funcionaría como un ancla a los salarios tanto en períodos de recesión como de expansión. En recesión ayudaría a mantener la presión deflacionaria sobre la economía suavizando la caída de la demanda, al mantener los ingresos de los trabajadores que son expulsados del sector privado; mientras que en expansión, como alternativa a las demandas salariales de los trabajadores del sector privado, los empleadores privados podrían reclutar trabajadores de la reserva o fondo que es el PTG simplemente atrayéndolos con unas mejores condiciones contractuales a las establecidas por el sector público, evitando así las presiones salariales que dan lugar a una espiral precios-salarios (Wray, 2009). Con base en su funcionamiento como estabilizador del ciclo, es fácil advertir que el tamaño de este plan vendrá impulsado por las decisiones de inversión corporativa. 
De acuerdo con Mosler (1997-1998), el PTG se puede considerar una política monetaria basada en un estándar salarial del trabajo, en lugar de un patrón oro. Si el Gobierno limita el salario monetario que paga a los trabajadores de los programas de garantía de empleo incluidos en el plan en lugar de restringir la cantidad de dinero gastada (como ocurre en el proceso presupuestario habitual), el efecto resultante sería la estabilidad de precios, utilizándose la mano de obra como un producto en un sistema de almacenaje regulador gestionado por el Gobierno de manera anticíclica.

Debe valorarse que este mecanismo estabilizador tiene adicionalmente un efecto positivo sobre la productividad del capital humano, gestionando el continuo cambio estructural y el avance tecnológico sin perjudicar la necesaria flexibilidad del sistema para que las empresas gestionen su capacidad productiva atendiendo a las presiones competitivas. Tal y como argumenta Forstater (1998), el PTG funcionaría como un ejército de reserva de trabajadores, pero con trabajadores que se moverían entre empleos del sector público y del sector privado y no entre una situación de empleo y otra de desempleo, posibilitando una mayor flexibilidad funcional, al mantener y mejorar sus habilidades, al margen de que se alcanzaría también la plena ocupación sin mercados de trabajo demasiado rígidos.

En definitiva, el chartalismo y la hacienda funcional muestran cómo los déficits públicos pueden ser utilizados como herramientas para lograr que la economía funcione logrando el pleno empleo con estabilidad de precios. Operativamente esto es posible, pero un objetivo más amplio de lo que Kaboub (2007) define como «ajuste institucional» es concretar qué tipo de pleno empleo queremos. A qué actividades va a destinarse este gasto público va a determinar el tipo de sociedad que queremos construir, y ello requiere una evaluación y diseño más profundo de las políticas públicas.

Ya hemos comentado que el PTG como herramienta de política económica difiere de la idea estándar de subvencionar los beneficios. Dicha idea se refiere a la estrategia de cerrar la brecha entre la producción actual y potencial mediante el impulso del gasto privado a partir de deducciones y créditos fiscales, contratos con beneficios garantizados y otras políticas que favorecen directamente a los ingresos del capital sin crear nunca las oportunidades de empleo suficientes para todos los individuos capaces y dispuestos a trabajar. Muy al contrario, los programas de garantía de empleo se alejan de la noción de «redistribución» que se utiliza habitualmente para describir el propósito público y permiten una conceptualización crítica del mismo, posibilitando lo que Keynes describió como socialización de la inversión (Minsky, 2008a: 154-157). No se trata solo de activar la economía a través del sector público, sino de repensar el papel de este.

Al hilo de lo anterior, Todorova (2013) propone enlazar el PTG con el concepto de aprovisionamiento social, basado en un proceso que abarca 
actividades no mercantiles, el cual incluye la actividad tanto productiva como reproductiva y los ecosistemas donde tiene lugar la actividad mercantil, enmarcándose en un contexto cultural e histórico. El proceso de aprovisionamiento social lo podemos definir como el estudio de cómo atender las necesidades de los integrantes de una sociedad, integrando la problemática de las relaciones de poder, las instituciones y el carácter inestable de la dinámica capitalista.

Por su parte, y desde una óptica schumpeteriana, Mazzucato (2014) presenta al sector público como un agente principal en la construcción creativa. En lugar de limitar su desempeño a las intervenciones que tradicionalmente le son concedidas (reducir el riesgo para el sector privado y corregir los denominados fallos de mercado o externalidades), el Estado no solo debe incentivar la inversión privada, sino también dinamizarla asumiendo la incertidumbre radical, moldeando y creando nuevos mercados, o creando las condiciones necesarias para la innovación. Esto exige alejarse de la visión del Estado como enemigo de la empresa privada y avanzar hacia una etapa donde se produzca un desarrollo del capital que sea resultado de la simbiosis entre el sector público y el sector privado. En conclusión, un sector público pasivo que subsidia los beneficios privados deseados a través de la demanda puede ser sustituido por un sector público emprendedor que lidere las iniciativas tecnológicas (Mazzucato y Wray, 2015; Medina, 2016: 127-135).

En nuestro criterio, repensar el papel del sector público a través de las nociones de aprovisionamiento social y del Estado emprendedor sirve como base estructural para orientar el diseño de los programas de garantía de empleo hacia la sostenibilidad ambiental y el desarrollo de las capacidades individuales. Siguiendo tal conceptualización del propósito público, dos pilares esenciales emergen en el diseño de un PTG: la preservación de la base ecológica de la actividad económica y la interconexión de la esfera productiva y la reproductiva.

Como expone Forstater (2003), es fácil imaginar cómo satisfacer las condiciones biofísicas para una economía sostenible sin garantizar el aprovisionamiento material necesario, simplemente obligando al cese de todas las actividades productivas. Esto dista de ser una solución aceptable. Dado que los programas de garantía de empleo no están sujetos a la lógica del lucro y la eficiencia económica sometida a la reducción de costes no es una preocupación, un PTG puede utilizar métodos de producción o fuentes de suministro energético renovables o que tengan un impacto ambiental lo menor posible. Pero además, las actividades de estos programas pueden destinarse a proyectos de investigación en energías alternativas, sorteando la exigencia de beneficios a corto plazo y la huida de los inversores en cuanto estos no se producen. Otros ejemplos de actividades que se podrían desempeñar para promover la sostenibilidad, y que son apuntados por Forstater, son: el reciclaje, la eficiencia 
energética de las infraestructuras públicas y hogares, la reducción del uso del automóvil, la utilización de la energía solar en los elementos del mobiliario urbano, la creación de jardines y huertos urbanos, la protección, seguridad y limpieza del medio ambiente, o la información, difusión y educación en sostenibilidad ambiental.

El otro pilar de un PTG que mencionábamos anteriormente es el referente a las actividades de cuidados, a la miríada de actividades que sostienen nuestro proceso vital. Un programa de garantía de empleo que abarque estos trabajos, generalmente no remunerados y que recaen habitualmente en las mujeres, permitiría a su vez transformar las relaciones de género. Como sugiere Antonopoulos (2007), la visibilización y valorización de este tipo de empleos podría iniciar un proceso de transformación de las relaciones de género en los hogares y las comunidades, especialmente si los hombres comienzan a participar en estas actividades de cuidado, ahora remuneradas. Todorova (2009) advierte de que ello no implica la mercantilización de la esfera reproductiva, sino la socialización del trabajo de cuidados, apreciación con la que coincidimos.

Por último, cabe afirmar que el diseño e implementación de los programas de garantía de empleo estarían completamente descentralizados, siendo la fuente de su financiación la única característica centralizada. De esta forma, y como indica Kaboub (2008), un PTG podría articularse de manera que los grupos locales de la comunidad, sindicatos y organizaciones sin ánimo de lucro examinaran las necesidades existentes a nivel local, pudiendo contratar a trabajadores para prestar dichos servicios como parte de los programas de garantía de empleo. Naturalmente, los trabajadores de estos programas solo producirían servicios que no son suministrados por el sector privado ni son realizados por funcionarios. Se trataría de empleos de transición, para los cuales no siempre se necesitaría una formación específica, sin perjuicio de constituir trabajos necesarios y útiles para la sociedad.

\section{CONCLUSIONES: HACIA UN MARCO INSTITUCIONAL OPERATIVO}

Una de las ideas centrales que subyacen de las páginas anteriores es el hecho de que una nación monetariamente soberana siempre tiene capacidad de financiar sus programas públicos. En un marco institucional donde estén consolidados el banco central y el tesoro como brazos monetario y fiscal del Estado, las operaciones de gasto constituyen un abono en las cuentas bancarias privadas, destruyéndose dinero a través de los impuestos al generarse un débito en ellas. 
Lo anterior no significa, sin embargo, que la recaudación de impuestos no sea importante, puesto que la capacidad de imposición es crucial para mantener la aceptación y circulación de la moneda a través de su demanda, que de otro modo no tendría respaldo. De hecho, en las economías de producción monetaria el valor de la moneda se vincula a lo que se debe hacer para obtenerla, y el sector público puede establecer directamente sus términos de intercambio con la compra de trabajo a través de los programas de garantía de empleo. La fiscalidad sería, en este sentido, la forma en que la autoridad se hace con bienes y servicios del sector privado, esto es, la forma de movilizar recursos privados al dominio público, sin que ello tenga nada que ver con la necesidad de ingresar dinero para financiar gasto alguno, pues, como hemos visto, esto es lógicamente inconsistente con el hecho fehaciente de que la autoridad siempre gasta primero.

En esta lógica, el déficit público constituye necesariamente un superávit del sector privado, en lo que a tenencia de activos financieros netos se refiere. Si el sector no gubernamental desea crónicamente ahorrar más de lo que invierte, el resultado será una creciente brecha de demanda que no puede ser cubierta por otros agentes del sector privado. De manera que el desempleo sería la evidencia de facto de que el déficit del Gobierno es demasiado bajo como para proporcionar el nivel de ahorro neto deseado.

La reflexión anterior suscita una cierta responsabilidad institucional de los Gobiernos en la generación y mantenimiento del desempleo, la cual derivaría en parte del hecho de que, a pesar de regir en las economías capitalistas actuales un moderno sistema de dinero fiduciario-crédito, las autoridades fundamentan sus decisiones en normas y preceptos propios de un enfoque metalista, tal y como acontece de forma evidente en la actual UEM.

Como explica Mitchell (2015), los Estados que han adoptado el euro han renunciado a su soberanía monetaria y, por extensión, a las capacidades intrínsecas de la política fiscal. Con esta arquitectura institucional se vulnera la enseñanza esencial de la teoría chartalista, que expone que el dinero es una criatura del Estado, cerrándose así el paso al funcionamiento de un marco operativo basado en las finanzas funcionales, e imponiéndose consecuentemente en el terreno de las políticas económicas la austeridad y los objetivos de equilibrio presupuestario que caracterizan a las, paradójicamente, denominadas finanzas sanas.

Las consecuencias de la separación de los poderes fiscales y monetarios como defecto principal de la configuración de la Zona Euro fueron profetizadas por Godley (2012) y Parguez (1999). Al perder la facultad de emitir su propio dinero y hacer giros sobre sus propios bancos centrales, los Gobiernos debían comprometerse a cumplir con la restricción presupuestaria para encontrar financiación en los mercados. Inevitablemente, mientras los agentes privados estaban 
en la etapa alcista del ciclo económico, endeudándose para aprovechar las oportunidades que ofrecían unas expectativas optimistas, el sector público conseguía tener superávits presupuestarios. Pero cuando las expectativas cambiaron y el sector privado se introdujo en un proceso de desapalancamiento, la consecución de superávits del sector público en un escenario de recortes del gasto público no solo constituyó un imposible, sino que fue, además, manifiestamente contraproducente. Tal ceguera en torno a las relaciones de los balances de los agentes impuso, de hecho, un sesgo deflacionario a la economía, llevándola a la depresión ${ }^{8}$.

En nuestro criterio, la teoría chartalista y la hacienda funcional muestran cómo los déficits públicos pueden ser utilizados como herramientas para lograr que la economía funcione con pleno empleo. También enseñan cómo el PTG puede servir para anclar el resto de precios de la economía al salario ofrecido, integrando un potente estabilizador de los precios, además de ser un eficaz estabilizador del ciclo sin disminuir la flexibilidad del sistema, llegando incluso a aumentarla gracias al aumento de la productividad de los trabajadores derivado de la diferencia de capacidades existente entre una situación de desempleo y otra de actividad en un PTG. Además, los programas de garantía de empleo pueden igualmente diseñarse para lograr objetivos centrados en el aprovisionamiento social y el impulso de la innovación, en tanto que pueden enfocarse hacia el cambio de modelo energético, el cuidado del medio ambiente o la atención a las personas dependientes.

En definitiva, la ruptura del nexo natural entre la política monetaria y la política fiscal constituye un fallo institucional. Abordar la relación entre los balances sectoriales en un esquema de flujos y stocks, tomar en consideración cuál es la función del dinero y los déficits públicos en la economía, y replantearse la posición de independencia del banco central y el papel del Estado en la economía son cuestiones que los investigadores macroeconómicos deben afrontar e integrar en sus modelos si de verdad quieren proveer soluciones a los problemas reales. Ello obliga también, necesariamente, a un profundo análisis de las instituciones, cuyos fundamentos han sido ignorados ante el predominio del individualismo metodológico. Esto ha ocurrido especialmente en el marco de la política monetaria, en que las ficciones de la teoría metalista han relegado al ostracismo cualquier planteamiento de carácter institucional, alejando del centro de discusión, incluso, el bienestar

8 Bellofiore (2013), desde una posición marxista, y Lavoie (2015), desde una óptica keynesiana, han señalado la configuración y las restricciones autoimpuestas del BCE como causante de la crisis del euro, un marco institucional disfuncional que, a criterio de estos autores, se autodestruye y terminará por romperse. 
general de los individuos. Ante esto, urge un viraje radical de la «economía política»; o lo que es igual, apremia la necesidad de «repensar la Economía», como han sugerido, entre otros autores, Blanchard, Dell'Ariccia y Mauro (2010). Y en todo ello, la teoría monetaria moderna es uno de los caminos posibles a explorar.

\section{Bibliografía}

Antonopoulos, R. (2007). The right to a job, the right types of projects: employment guarantee policies from a gender perspective. Levy Economic Institute Workings Paper, 516. Disponible en: https://doi.org/10.2139/ssrn.1016197.

Bell, S. (2000). Do taxes and bonds finance government spending? Journal of Economic Issues, 34 (3), 603-620. Disponible en: https://doi.org/10.1080/00213624.2000.11506296.

- (2001). The role of the state and the hierarchy of money. Cambridge Journal of Economics, 25 (2), 149-163. Disponible en: https://doi.org/10.1093/cje/25.2.149.

Bell, S. y Henry, J. F. (2001). Hospitality versus exchange: the limits of monetary economies. Review of Social Economy, 59 (2), 203-226. Disponible en: https://doi. org/10.1080/00346760110036166.

Bellofiore, R. (2013). "Two or three things I know about her": Europe in the global crisis and heterodox economics. Cambridge Journal of Economics, 37 (3), 497-512. Disponible en: https://doi.org/10.1093/cje/bet002.

Blanchard, O., Dell'Ariccia, G. y Mauro, P. (2010). Rethinking macroeconomic policy. Journal of Money, Credit and Banking, 42 (s1), 199-215. Disponible en: https://doi. org/10.1111/j.1538-4616.2010.00334.x.

Cesaratto, S. (2016). The state spends first: Logic, facts, fictions, open questions. Journal of Post Keynesian Economics, 39 (1), 44-71. Disponible en: https://doi.org/10.1080/01603 477.2016.1147333.

Cruz, E. y Parejo, F. M. (2016). El dinero en la historia del pensamiento económico: la teoría monetaria post-keynesiana y su confrontación con la ortodoxia. Iberian Journal of the History of Economic Thought, 3 (1), 27-41.

Espósito L. y Mastromatteo, G. (2015). The Two Approaches to Money: Debt, Central Banks, and Functional Finance. Levy Economics Institute, Working Paper, 855.

Forstater, M. (1998). Flexible full employment: structural implications of discretionary public sector employment. Journal of Economic Issues, 32 (2), 557-563. Disponible en: https:// doi.org/10.1080/00213624.1998.11506064.

- (1999). Functional finance and full employment: lessons from Lerner for today. Journal of Economic Issues, 33 (2), 475-482. Disponible en: https://doi.org/10.1080/00213624 .1999 .11506180 .

(2003). Public employment and environmental sustainability. Journal of Post Keynesian Economics, 25 (3), 385-406.

Fullwiler, S. T., Kelton, S. A. y Wray L. R. (2012). Modern Money Theory: A Response to Critics. Political Economy Research Institute Working Papers, 279. Disponible en: https://doi. org/10.2139/ssrn.2008542. 
Godley, W. (2012). Maastricht and All That. En M. Lavoie y G. Zezza (eds.). The Stock-flow Consistent Approach: Selected Writings of Wynne Godley. Basingstoke: Palgrave Macmillan. Disponible en: https://doi.org/10.1057/9780230353848_9.

Graeber, D. (2012). En deuda. Una historia alternativa de la economia. Barcelona: Ariel.

Henry, J. F. (2004). The Social Origins of Money: The Case of Egypt. En L. R Wray (ed.). Credit and State Theories of Money (pp. 79-98). Cheltenham: Edward Elgar. Disponible en: https://doi.org/10.4337/9781843769842.00009.

Hudson, M. (2004a). The Archaeology of Money: Debt versus Barter Theories of Money's Origins. En L. R Wray (ed.). Credit and State Theories of Money (pp. 99-127). Cheltenham: Edward Elgar. Disponible en: https://doi.org/10.4337/9781843769842.00010.

- (2004b). The development of money-of-account in sumer's temples. En M. Hudson y C. Wuncsch (eds.). Creating Economic Order: Record-Keeping, Standardization, and the Developments of Accounting in the Ancient Near East (pp. 303-329). Maryland: CDL Press.

Ingham, G. (2000). "Babylonian madness"': On the historical and sociological origins of money. En J. Smithin (ed.). What Is Money? (pp. 16-41). London, New York: Routledge.

- (2004a). The nature of money. Cambridge: Malden Mass; Polity Press.

- (2004b). The Emergence of Capitalist Credit Money. En L. R. Wray (ed.). Credit and State Theories of Money (pp. 173-222). Cheltenham: Edward Elgar. Disponible en: https://doi.org/10.4337/9781843769842.00012.

Innes, A. M. (2004a). What is money. En L. R. Wray (ed.). Credit and State Theories of Money (pp. 14-49). Cheltenham: Edward Elgar. Disponible en: https://doi.org/10.4337/9781 843769842.00007 .

- (2004b). The Credit Theory of Money. En L. R. Wray (ed.). Credit and State Theories of Money (pp. 50-78). Cheltenham: Edward Elgar. Disponible en: https://doi.org/10.433 7/9781843769842.00008.

Juniper, J. y Mitchell, W. (2008). There is no financial crisis so deep that cannot be dealt with by public spending. Newcastle: Centre of Full Employment and Equity.

Juniper, J., Sharpe, T. P. y Watts, M. J. (2015). Modern monetary theory: Contributions and critics. Journal of Post Keynesian Economics, 37 (2), 281-307.

Kaboub, F. (2007). Institutional adjustment planning for full employment. Journal of Economic Issues, 41 (2), 495-502. Disponible en: https://doi.org/10.1080/00213624.2007.1 1507038.

- (2008). Elements of a radical counter-movement to neoliberalism: Employment-led development. Review of Radical Political Economics, 40 (3), 220-227. Disponible en: https://doi.org/10.1177/0486613408319918.

Kiyotaky, N. y Wright, R. (1989). On money as a medium of Exchange. Journal of Political economy, 97 (4), 927-954. Disponible en: https://doi.org/10.1086/261634.

Knapp, G. F. (1924). The State Theory of Money. London: Macmillan and Company Limited. Lavoie, M. (2013). The monetary and fiscal nexus of neo-chartalism: A friendly critique. Journal of Economic Issues, 47 (1), 1-32. Disponible en: https://doi.org/10.2753/JEI00213624470101.

- (2015). The Eurozone: Similarities to and differences from Keynes's plan. International Journal of Political Economy, 44 (1), 3-17. Disponible en: https://doi.org/10.1080/089 11916.2015.1035980. 
Lerner, A. P. (1943). Functional finance and the federal debt. Social Research, 10, 38-51.

- (1957). Economía del pleno empleo. Madrid: Aguilar Ediciones.

Mazzucatto, M. (2014). El Estado emprendedor. Barcelona: RBA.

Mazzucatto, M. y Wray, L. R. (2015). Financing the capital development of the Economy: a Keynes-Schumpeter-Minsky synthesis. Laboratory of Economics and Management Papers Series, 2015/14. Disponible en: https://doi.org/10.2139/ssrn.2603847.

Medina, S. (2016). El leviatán desencadenado. Berlín: Lola Books.

Menger, K. (1892). On the origin of money. The Economic Journal, 2 (6), 239-255. Disponible en: https://doi.org/10.2307/2956146.

Minsky, H. (2008a). John Maynard Keynes. New York: McGraw-Hill.

- (2008b). Stabilizing an unstable economy. New York: McGraw-Hill.

Mitchell, W. (1998). The buffer stock employment model and the NAIRU: The path to full employment. Journal of Economic Issues, 32 (2), 547-555. Disponible en: https://doi.org /10.1080/00213624.1998.11506063. (2015 [2016]). La distopia del euro. Berlín: Lola Books.

Mitchell, W. y Mosler, W. (2002). Fiscal policy and the job guarantee. Australian Journal of Labour Economics, 5 (2), 243-259.

Mitchell, W. y Wray, L. R. (2005). In defense of employer of last resort: a response to Malcolm Sawyer. Journal of Economic Issues, 39 (1), 235-244. Disponible en: https://doi.org/10.1 080/00213624.2005.11506788.

Mosler, W. (1993). Soft Currency Economics II: The Origin of Modern Monetary Theory. Createspace Independent Publishing Platform.

- (1997-1998). Full employment and price stability. Journal of Post Keynesian Economics, 20 (2), 167-182. Disponible en: https://doi.org/10.1080/01603477.1997.11490146.

Nersisyan, Y. y Wray, L. R. (2016). Modern Money Theory and the facts of experience. Cambridge Journal of Economics, 40 (5), 1297-1316. Disponible en: https://doi.org/10.1093/ cje/bew015.

Nesiba, R. F. (2013). Do Institutionalists and post-Keynesians share a common approach to Modern Monetary Theory (MMT)? European Journal of Economics and Economic Policies, 10 (1), 44-60. Disponible en: https://doi.org/10.4337/ejeep.2013.01.05.

Papadopoulos, G. (2013). Money and value: a synthesis of the state theory of money and original institutional economics. The Journal of Philosophical Economics, 6 (2), 2-22.

Parguez, A. (1999). The expected failure of the European Economic and Monetary Union: A false money against the real economy. Eastern Economic Journal, 25 (1), 63-76.

Polanyi, K. (1976a). El sistema económico como proceso institucionalizado. En M. Godelier (ed.). Antropología y Economía. Barcelona: Anagrama.

(1976b). Intercambio sin mercado en tiempos de Hammurabi. En K. Polanyi, C. M. Arensberg y H. W. Pearson (eds.). Comercio y mercado en los imperios antiguos. Barcelona: Labor Universitaria.

Tcherneva, P. (2002). Monopoly Money: The State as a Price Setter. Oeconomicus, 5, 30-52.

— (2012). Permanent on-the-spot job creation. The missing Keynes Plan for full employment and economic transformation. Review of Social Economy, 70 (1), 57-80. Disponible en: https://doi.org/10.1080/00346764.2011.577348. 
- (2014). Reorienting fiscal policy: A bottom-up approach. Journal of Post Keynesian Economics, 37 (1), 43-66. Disponible en: https://doi.org/10.2753/PKE0160-3477370105.

Todorova, Z. (2009). Employer of Last Resort Policy and Feminist Economics: Social Provisioning and Socialization of Investment. Center for Full Employment and Price Stability Working Paper Series, 56. Disponible en: https://doi.org/10.2139/ssrn.1438165.

- (2013). Connecting social provisioning and functional finance in a post-Keynesian-Institutional analysis of the public sector. European Journal of Economics and Economic Policies: Intervention, 10 (1), 61-75.

Tymoigne, É. (2016). Government monetary and fiscal operations: generalising the endogenous money approach. Cambridge Journal of Economics, 40 (5), 1317-1332. Disponible en: https://doi.org/10.1093/cje/bew012.

Tymoigne, É. y Wray, L. R. (2006). Money: an alternative story. En P. Arestis y M. Sawyer (eds.). A Handbook of Alternative Monetary Economics. Cheltenham: Edward Elgar. (2013). Modern Money Theory 101: A Reply to Critics. Levy Economics Institute of Bard College Working Papers, 778. Disponible en: https://doi.org/10.2139/ssrn.2348704.

Wray, L. R. (1998). Understanding modern money. Cheltenham: Edward Elgar.

- (2000a). Modern Money. En J. Smithin (ed.). What Is Money? (pp. 42-47) London, New York: Routledge.

- (2000b). The Employer of Last Resort Approach to Full Employment. Center for Full Employment and Price Stability Working Paper, 9.

- (2009). An alternative view of finance, saving, deficits, and liquidity. International Journal of Political Economy, 38 (4), 25-43. Disponible en: https://doi.org/10.2753/ IJP0891-1916380402.

- (2014). From the state theory of money to modern money theory: An alternative to economic orthodoxy. Levy Economics Institute Working Papers Series, 792. Disponible en: https:// doi.org/10.2139/ssrn.2407711.

- (2015). Teoría monetaria moderna: manual de macroeconomía sobre los sistemas monetarios soberanos. Berlín: Lola Books. 